\title{
Variations
}

Variations

Revue internationale de théorie critique

$8 \mid 2006$

Subjectivités libres et critique de la répression

\section{Contre la précarité et la subordination, construire le dépassement du salariat par de nouvelles formes de gestion}

\section{Philippe Maingault}

\section{OpenEdition}

\section{Journals}

Édition électronique

URL : http://journals.openedition.org/variations/511

DOI : 10.4000/variations. 511

ISSN : 1968-3960

Éditeur

Les amis de Variations

\section{Édition imprimée}

Date de publication : 1 novembre 2006

Pagination : 90-100

Référence électronique

Philippe Maingault, «Contre la précarité et la subordination, construire le dépassement du salariat par de nouvelles formes de gestion », Variations [En ligne], 8 | 2006, mis en ligne le 27 décembre 2012, consulté le 01 mai 2019. URL : http://journals.openedition.org/variations/511 ; DOI : 10.4000/ variations. 511

Ce document a été généré automatiquement le 1 mai 2019.

Les ami•e•s de Variations 


\title{
Contre la précarité et la subordination, construire le dépassement du salariat par de nouvelles formes de gestion
}

\author{
Philippe Maingault
}

Dans la lutte contre le CPE, le contrat à durée indéterminée (CDI) a souvent été idéalisé dans les discours associatifs, syndicaux ou politiques de gauche. La revendication « d'un CDI pour tous » a été massivement reprise et, dans le contexte de détérioration de la condition salariale, elle est apparue comme une situation quasiment idéale pour les travailleurs. Pourtant, la plupart des salariés français sont encore couverts par des $\mathrm{CDI}^{1}$, sans que leur sort soit toujours enviable. Salaires insuffisants, peur du chômage, mal-être et souffrance au travail, maladies professionnelles, sentiment de routine, stress, ou encore temps partiel imposé, caractérisent souvent la condition salariale, fût-elle sous forme de CDI.

\section{Le CDI et ses limites}

2 Si le CDI est en effet plus protecteur pour les salariés, il n'en reste pas moins que celui-ci maintient l'employé subordonné dans la relation de travail. Le contrat de travail était auparavant appelé "contrat de louage de service », et cette terminologie se juxtaposa avec celle de contrat de travail jusqu'à ce que la loi 73-4 du 2 janvier 1973 sur le licenciement ne vienne prévoir que désormais, seul le terme de contrat de travail serait utilisé.

3 Si les textes de loi ne définissent pas le contrat de travail en tant que tel, la jurisprudence le caractérise : c'est une "convention par laquelle une personne physique s'engage à mettre son activité à la disposition d'une autre personne, physique ou morale, sous la subordination de laquelle elle se place, moyennant une rémunération²." 
Évidemment, ce lien de subordination est quelque peu amoindri par l'existence du droit du travail. En l'absence d'un tel droit, le contrat entre les deux parties (employeur et employé) serait davantage soumis aux rapports de force économique. Imaginons l'absence de droit spécifique du travail dans les secteurs qui licencient ou dans ceux où la demande de travail est pléthorique. Les vacances seraient supprimées, le temps de travail considérable, le droit à la maternité interdit la plupart du temps... Pour limiter l'inégalité contractuelle, le mouvement ouvrier, les mouvements sociaux, se sont battus tout au long de l'Histoire pour conquérir un droit du travail diminuant en quelque sorte l'inégalité entre les parties, détachant le rapport salarial du simple lien contractuel du droit civil.

5 Ainsi, un salarié en CDI est dans une position moins délicate qu'un salarié en contrat à durée déterminée (CDD) ou en contrat nouvelle embauche (CNE). Mais il reste subordonné et à la merci de l'arbitraire patronal, surtout dans les petites entreprises, où le rapport de force, l'inégalité contractuelle est la plus forte. Dans les grandes entreprises, là où existent généralement des syndicats, l'inégalité contractuelle est moins forte, car le CDI légal peut être amélioré par des conventions collectives de branche plus favorables.

Des CDI et des garanties collectives assez puissantes dans les grandes entreprises ont existé notamment dans la période dite des « 30 glorieuses». Cette situation, un peu mythifiée aujourd'hui, n'était pourtant qu'un compromis de type keynésien, entre le capital contraint d'accepter certaines règles limitant sa toute-puissance, et un salariat ayant réussi à diminuer l'insécurité sociale sans pour autant devenir maître de son outil de travail et donc de son destin. Souvenons-nous des Temps modernes de Chaplin, œuvre majeure illustrant la dépossession et l'instrumentalisation du travailleur !

7 Aujourd'hui, précarisation et insécurité sociale grandissantes nous feraient parfois presque regretter cette époque. En effet, la précarisation et le chômage accroissent la subordination salariale. En même temps, l'exigence actuelle d'émancipation nécessite de viser plus loin que le retour à un compromis salarial modernisé. L'époque appelle un dépassement du salariat, à une sortie du lien de subordination qui lui est consubstantiel. Le simple retour en arrière n'est pas une solution et ne correspond pas aux aspirations profondes du salariat. Le besoin d'épanouissement au travail, de maîtrise de son destin, se traduit, notamment parmi la jeunesse, par le désintérêt croissant d'un travail balisé, routinier jusqu'à la retraite. Comment comprendre sinon que les démissions du CDI sont relativement fréquentes chez les jeunes gens, alors même que le marché du travail leur est défavorable? André Gorz ${ }^{3}$ souligne d'ailleurs, en évoquant les emplois stables, que « chez les moins de 35 ans la majorité ne désire plus ce genre d'emploi, préférant une vie multidimensionnelle, multiactive, polycentrique...» Comment comprendre le désir fort de s'investir dans les organisations humanitaires, dans les activités équitables, ou de créer sa propre entreprise, alors même que ces différentes voies professionnelles ne sont pas les plus rémunératrices ou les plus assurées? Doit-on interpréter ces comportements comme relevant de l'instabilité ou n'existe-t-il pas une quête - pas toujours explicite d'autre chose, c'est-à-dire d'autonomie, d'épanouissement, de dépassement de la marchandisation de chacun?

Cette recherche d'autres rapports au travail peut s'expliquer par la qualification croissante des jeunes, qualification débouchant sur une autonomie accrue de travail, de pensée, d'organisation. Cela contredit les organisations hiérarchiques et dissymétriques du monde salarial. Le besoin d'affirmation de sa singularité croit, sans pour autant que cela ne signifie nécessairement individualisme, mise en compétition. Or, cette autonomie est brisée par le salariat et ses avatars. En même temps, le dépassement progressiste du salariat semble inatteignable. La sortie du capitalisme 
est souvent considérée comme un préalable au dépassement des rapports sociaux qu'il a instaurés, du moins pour ceux qui considèrent encore comme étant nécessaire d'instaurer d'autres rapports au travail. En effet, de nombreuses forces limitent leurs ambitions à une sécurisation de la condition salariale. Ces forces politiques et syndicales envisagent le dépassement du capitalisme, mais sans remettre fondamentalement en cause le rapport salarial, bien que des expériences similaires aient été tentées dans les pays d'Europe de l'Est, sans être concluantes. Les salariés, sécurisés dans leur vie et au travail, étaient formellement propriétaires individuels des outils de travail de leur Nation. Mais cette propriété n'a existé qu'en théorie, dans la mesure où la maittrise effective de l'outil de production et des échanges échappait aux travailleurs, au profit de mécanismes anonymes et technocratiques de planification, plus ou moins centralisés. Le maintien du salariat a certainement contribué au désintérêt et à la déresponsabilisation au travail.

Il convient donc d'imaginer des voies différentes pour éviter de nouveaux écueils. Avant cela, nous voudrions exposer et soumettre à l'analyse critique une série de propositions déjà existantes, parmi lesquelles certaines sont censées améliorer voire dépasser le salariat.

\section{Sorties régressives du salariat}

La sortie du salariat peut être régressive. C'est la forme souvent imposée par le patronat à travers des contrats d'externalisation, de sous-traitance, des systèmes de franchises, de diverses formes de travail « indépendant $» . .$.

11 Sous l'apparence d'une autonomie plus grande par rapport au patronat, la réalité cache souvent des rapports de domination et de dépendance exacerbés non bénéfiques à ces travailleurs captifs, dits «indépendants ». Les expériences de participation des salariés via diverses formules au capital des entreprises relèvent globalement d'une mystification, lourde de dangers, car l'asymétrie d'information favorise le patronat et les actionnaires principaux, au détriment des salariés-actionnaires. Les scandales financiers qui émaillent périodiquement les colonnes des journaux révèlent l'ampleur vraisemblablement croissante des délits d'initiés. La complexification grandissante des systèmes sociotechniques multiplie les besoins d'investissement et d'équipements lourds. La rentabilisation du capital investi devient plus aléatoire et renforce les tentations de triche au détriment des actionnaires minoritaires, moins bien informés.

Les économistes libéraux, dont notamment Milton Friedman, prônent aussi l'impôt négatif afin d'augmenter les ressources des salariés les plus pauvres et d'inciter les chômeurs à reprendre un travail, devenu peu attractif par des salaires trop faibles. Des dispositifs d'inspiration comparable ont déjà existé dans le passé. Ainsi, le système Speenhamland, introduit en Angleterre à la fin du XVIIIe siècle a institué, pour combler le déficit salarial, un supplément de rémunération assurant au salarié un revenu minimum indexé sur le prix du pain.

13 Ce mécanisme, en place en France depuis 2000 avec la prime pour l'emploi, s'apparente en fait à un report sur la société du paiement d'une partie du salaire et donc à une diminution du prix d'achat du travail. Indirectement, cette prime équivaut à déclarer : « Votre travail n'a pas assez de valeur pour qu'il soit rémunéré correctement. Vous êtes donc en partie à la charge de la société. Ne vous plaignez donc pas!» Cela revient à culpabiliser les personnes concernées. 
14 En effet, l'assistance ne crée pas l'irresponsabilité, contrairement au discours dominant, mais surtout l'assujettissement via la culpabilisation.

\section{Le revenu d'existence et ses contradictions}

15 De la même façon, les revenus d'existence, type RMI, ou même les revendications d'allocation universelle (revenu moins indigent que le RMI car universel, sans aucune conditionnalité et cumulable avec d'autres ressources), souvent présentés comme sortie possible de la dépendance salariale, constituent de fausses alternatives. Le RMI s'insère bien dans les dispositifs libéraux, assez proches de l'allocation complémentaire ou de l'impôt négatif, en ce sens que l'insuffisance de valeur marchande d'une partie de la force de travail rend celle-ci dépendante de la "générosité" publique. Générosité indispensable pour assurer une relative paix sociale et civile dans des pays où la misère côtoie le luxe le plus débridé.

L'allocation universelle ou revenu garanti émane de secteurs progressistes de la société. Cette allocation universelle s'appuie notamment sur l'idée selon laquelle la puissance de la force de travail réside de plus en plus dans le savoir accumulé en amont du moment traditionnel proprement productif. Cependant, cette théorie opère progressivement des glissements sémantiques et finit par considérer que «ce n'est plus le travail qui crée de la richesse, mais la science et la technique, le general intellect, dont le lieu n'est pas l'usine, mais la sociétét ${ }^{4}$. Michael Hardt et Toni Negri vont même écrire que "toutes les formes de pauvreté sont devenues productives ${ }^{5}$." La production immanente de la valeur détruirait la traditionnelle loi de la valeur et justifierait alors l'allocation universelle, dans une société où la localisation spatiale et temporelle de la création de valeur s'estomperait complètement. Cette dilution de la valeur dans la théorie du capitalisme cognitif justifie l'hypothèse " distributiviste ». Cette théorie présente un caractère quelque peu mystique. Car le savoir social ne devient productif que s'il est mobilisé ; le travail immatériel reste virtuel s'il n'est pas concrétisé par une dépense matérielle d'énergie réelle ${ }^{6}$.

valeur continue essentiellement à être produite dans des espaces spatio-temporels définissables, même si le cadre général (appareil de formation, savoir accumulé, réseaux de coopération) joue un rôle essentiel croissant dans l'efficacité productive. Même ce cadre général ne reste qu'une potentialité s'il n'est pas concrétisé par l'acte productif direct. Ce cadre productif, profondément reconfiguré, complexifie la mesure de la valeur. Faut-il pour autant abandonner toute velléité de mesure de la valeur, accepter une dilution de celle-ci conduisant à l'émergence de normes distributivistes niant la singularité des apports de travail de chacun?

Ce syncrétisme économique apparaît en outre contradictoire avec l'exigence croissante de reconnaissance de subjectivité des individus ; en cela, il est source de tensions sociales.

Par ailleurs, l'allocation universelle apparaît trop souvent comme un distributivisme « au rabais » laissant la place à d'immenses inégalités au-dessus du revenu minimum. Cela implique en définitive l'acceptation en creux d'une part de rémunération non liée à l'échange marchand pour les besoins fondamentaux, tandis que le solde de rémunération resterait fonction de la "capacité marchande » de chaque individu. Un distributivisme très ambitieux conduirait finalement à un égalitarisme peu respectueux des singularités de chacun et ne correspondant pas à l'état d'esprit de la plupart des sociétés. L'échec du « socialisme est européen » renvoie d'ailleurs fortement aux écueils du distributivisme. 

l'impasse sur l'éradication du chômage comme objectif de lutte. Derrière cette impasse, se profile vraisemblablement une surdétermination du potentiel productif généré par le travail immatériel et le savoir scientifique.

21 Si l'économie cognitive est susceptible de libérer un potentiel énorme de croissance, des facteurs puissants contrecarrent cette tendance. La crise écologique provoque tendanciellement des externalités négatives et croissantes. S'il était techniquement possible de comptabiliser lesdites externalités d'un point de vue monétaire, nous serions peut-être actuellement en phase de décroissance du PIB.

Surmonter la crise écologique nécessitera de diminuer la productivité dans un certain nombre de secteurs économiques (l'agriculture par exemple), afin d'y réduire les externalités environnementales négatives. Par ailleurs, dans le domaine des biens relationnels, les procédures automatiques et informationnelles ne peuvent être introduites comme élément de substitution du travailleur. La productivité actuelle d'un certain nombre de ces services est trop importante et est basée sur une charge trop lourde pour les travailleurs. La réduction de cette charge de travail, de cette productivité, ainsi que les mutations dans certains secteurs économiques sont producteurs d'emplois nouveaux. Par conséquent, il est possible de résorber le chômage par la création d'emplois. Celle-ci est absolument nécessaire au vu des millions de chômeurs dont le vœu le plus cher est de trouver un emploi, par besoin matériel mais aussi pour se socialiser, tisser des liens relationnels...

23 Si certains individus parviennent à tisser du lien social sans la médiation de l'emploi, la norme écrasante reste pour le moment, et certainement pour longtemps encore, la socialisation par l'emploi. On peut certes déplorer cette situation qui correspond à une culture issue de sociétés disciplinaires, fortement normatives, mais c'est ainsi, et se dégager de ces normes sera très long. Voici une des raisons majeures de l'inactualité du revenu garanti universel.

Pour autant les revenus d'existence, tels le RMI, ne sont pas à écarter dans la situation présente où des millions d'individus n'ont pas droit à un travail et à un revenu émanant de celui-ci. Ces solutions restent pourtant bancales et dangereuses, car elles dénient aux «bénéficiaires-victimes» toute capacité de participer à la vie économique, élément anthropologique de la socialité humaine. Elles doivent donc rester provisoires, d'autant plus que les formes distributivistes sont créatrices de conflits entre distributeurs et bénéficiaires d'allocations.

Le discours récurrent de la droite ${ }^{7}$ contre "l'assistanat, l'irresponsabilité » et donc le conditionnement des aides sociales aux personnes aptes, exploite particulièrement les failles de ces redistributions, d'ailleurs très modestes actuellement. Cette rhétorique souvent démagogique rencontre un écho parmi de larges couches salariées et favorise de ce fait la division des dominés.

Par contre, des systèmes d'assurance collective (retraites, maladie, handicap...) sont à développer et à étendre. On pourrait y adjoindre la sécurité sociale du logement avec une prise en charge collective du paiement des loyers ou le remboursement d'emprunts dans les moments d'accident de la vie (divorce, chômage tant qu'il ne sera pas éradiqué...), mais aussi le droit à une quotité d'eau et d'énergie minimale gratuite ${ }^{8}$. Cela n'aurait rien à voir avec l'assistance assujettissante du RMI, car ces droits-gratuités sont universels. Ils ne présentent pas non plus un des inconvénients de l'allocation sociale universelle qui 
monétarise et individualise des prestations fondamentales, déjà en partie gratuites et socialement bien acceptées. Ils ne visent pas non plus à une substitution de revenus du travail social.

Pour la jeunesse, une allocation d'autonomie ou revenu d'études pourrait par contre être versée à partir d'un certain âge (16 ou 18 ans par exemple) où l'autonomie par rapport aux parents est désirée.

\section{La sécurité d'emploi-formation (SEF) ou la sécurité sociale professionnelle (SSP)}

Pour couvrir le chômage, il existe actuellement des mécanismes assurantiels (l'assurancechômage), mais seule la moitié des chômeurs en bénéficie.

Paul Boccara, économiste communiste, a imaginé un système de sécurité d'emploi ou de formation pour dépasser le chômage et le marché du travail'9. La CGT a repris cette idée sous le vocable de "sécurité sociale professionnelle ». À présent, cette idée est récupérée en partie par le PS, mais aussi de façon dénaturée par une partie des libéraux. Le vocable généralement utilisé n'est plus "sécurité d'emploi-formation» ou "sécurité sociale professionnelle ", mais « sécurisation des parcours professionnels $»^{10}$. En quoi consiste cette SEF ou SSP ?

Il s'agit de créer un nouveau type de plein emploi non statique, en répondant aux besoins croissants de formation générés par la complexification continue des systèmes sociotechniques.

Dans cette optique, le salarié sorti de son entreprise ne se retrouverait plus chômeur, conserverait son contrat de travail et ses droits individuels acquis (rémunération, classification dans une grille salariale, couverture sociale). Ses droits individuels seraient transférables tout au long de sa carrière professionnelle. Cette dernière ne serait donc plus interrompue par la perte de l'emploi. Les moments professionnels entre deux emplois seraient notamment utilisés pour se former, afin de gagner en qualification et d'être ainsi plus efficace pour le nouvel emploi. Cette inter-période serait financée par des cotisations sociales (en premier lieu en diminuant les exonérations de «charges patronales ») mais aussi la taxation des revenus financiers.

Cette SEF/SSP représenterait évidemment un progrès très sensible par rapport à la situation présente. Le contrat de travail actuel, lien juridique entre deux parties inégales, que le droit légal et conventionnel du travail ne fait qu'atténuer, changerait de caractère grâce à cette sécurisation sociale, vecteur d'autonomie par rapport à l'employeur. En un sens, la SEF constituerait bien un dépassement du salariat, mais les choses ne sont pas si simples, et ce pour plusieurs raisons.

Premièrement, ce contrat nouveau peut devenir, dans le contexte économique d'aujourd'hui, un moyen de flexibilité à outrance et d'accompagnement social de restructurations économiques engendrées par la recherche de rentabilité du capital. L'abandon de garanties collectives et de certains mécanismes de protection contre le licenciement, au seul profit d'une individualisation des parcours, présente un risque. Jean-Louis Borloo plaide d'ailleurs pour des contrats de ce type, qu'il nomme «contrats transitionnels ». 
34 La «flex-sécurité » danoise, tant évoquée, constitue ce modèle dévoyé. Face à cela, il convient de maintenir et de renforcer le droit du licenciement collectif, notamment en interdisant les licenciements boursiers, visant uniquement à augmenter la rentabilité du capital.

Ces protections juridiques n'auront pas d'efficacité tangible sans modification de la vie économique. À cet effet, il convient de recréer de la stabilité via le développement de services publics puissants (de la Commune au Monde) et d'une économie sociale équitable pour ce qui ne relève pas du service public. Cette stabilité ne saurait signifier immobilité. $\mathrm{Au}$ contraire, la SEF/SSP pourrait être utilisée pour accompagner la nécessaire restructuration de nos modes de production et de consommation, de moins en moins créateurs de bien-être social et de plus en plus écologiquement destructeurs ${ }^{11}$. Ainsi, une diminution de la production automobile, corrélative d'une augmentation de la production de matériel de transports en commun, pourrait s'accompagner de périodes de formations puis de redéploiement d'effectifs.

Deuxièmement, il serait souhaitable d'enrichir la SEF/SSP en prenant en compte les aspirations montantes à d'autres occupations non professionnelles (congés sabbatiques pour des activités personnelles épanouissantes, détachements au profit d'occupations bénévoles...), voire à des formes atypiques de travail lorsqu'elles sont souhaitées.

Ce dépassement du salariat, via une SEF/SSP enrichie et non dévoyée, serait certes un grand progrès par rapport à la situation actuelle, mais il ne conduirait en définitive qu'à une sécurité individuelle, assez passive du travailleur. Passer du contrat salarial au statut salarial ne suffit pas à émanciper les travailleurs. D'ailleurs, le statut de fonctionnaire n'est pas forcément très éloigné de celui du salariat estampillé SEF/SSP. Cela suffit-il à faire du fonctionnaire un travailleur épanoui, émancipé ?

\section{Une nouvelle gestion par la maîtrise de ses outils de travail et d'échange}

Le second aspect de l'obsolescence du salariat réside dans la non-maîtrise des structures de travail par les principaux intéressés, les travailleurs. Maurice Décaillot ${ }^{12}$ souligne que ceux-ci ne maîtrisent ni l'utilisation de leurs moyens techniques, ni leurs échanges économiques. Ils restent ainsi dépendants de l'employeur. Pourtant, le travail contemporain et la part croissante de l'immatériel, de la coopération, appellent une mobilisation nouvelle de la subjectivité de chaque individu. Comment concilier ce besoin d'implication subjective avec le rapport salarial ? À côté d'une citoyenneté politique, une citoyenneté économique pourrait émerger, garantissant à chaque travailleur un rôle actif dans son activité économique. Décaillot explore des pistes intéressantes et propose le développement d'un système de producteurs-associés ${ }^{13}$, organisant non seulement de façon autonome leurs échanges économiques en amont et en aval de leur entreprise, mais maitrisant aussi techniquement l'utilisation de leurs propres outils de travail. Au lieu d'un salaire, les travailleurs recevraient un revenu d'activité, lié à l'efficience de leur travail. Ce système pourrait à première vue rappeler celui en vigueur autrefois dans l'autogestion yougoslave. Mais pour éviter la reproduction de rapports de force inégalitaires ${ }^{14}$ lors des échanges entre entités économiques autogestionnaires, Décaillot envisage de façon très astucieuse la construction de normes d'échanges équitables pour 
les biens connus et les services reproductibles, en prenant en compte l'apport en travail social de chaque entité économique participant à l'échange.

Afin de prévenir les risques d'égalitarisme dépossédant les individus et collectifs de la qualité de leur travail, des normes moyennes de productivité peuvent servir de référence pour l'échange entre les entreprises d'un même secteur. L'évaluation des biens courants ne pose pas de difficulté majeure, car les normes de production, les quantités produites et demandées sont relativement stables dans le temps. Pour les biens nouveaux, Décaillot ${ }^{15}$ propose une négociation entre échangistes avec procédures d'arbitrages par des tiers en cas de divergences sur la norme d'échanges. L'irruption accélérée de biens nouveaux complique cependant la réalisation d'un processus d'évaluation démocratique qui déboucherait pas à pas sur l'équité. On peut considérer que la construction d'une économie alternative réduira la multiplication intempestive, à grands renforts de publicité, de fausses innovations, de gadgets inutiles... Car une économie alternative devra combiner recherche d'épanouissement des individus et préservation des écosystèmes actuellement très menacés. Ceci conduira à une réduction des pseudonouveautés dont la seule utilité est la valorisation du capital; ainsi, l'irruption plus raisonnable de la nouveauté dans un contexte économique renouvelé diminuera la part des produits nouveaux à évaluer dans l'ensemble de la production.

Les services spécifiques peuvent entretenir, de leur côté, des rapports d'évaluation et d'échanges réputés équivalents avec le reste des activités économiques. La généralisation de ces rapports d'échange contractuels, soumis à l'arbitrage tiers en cas de désaccord, permettrait d'équilibrer de proche en proche les offres et demandes sans planification centrale omnisciente et dont l'efficacité est très incertaine. Cet "entreprenariat solidaire » nouveau pourrait émerger et constituer une alternative à la figure libérale de l'entrepreneur individuel «seul contre tous". La maitrise des outils de travail et d'échanges par les travailleurs-associés faciliterait, par ailleurs, le recul progressif de la division technique du travail, entre tâches routinières et particulières. Cette répartition dissymétrique du travail, poussé à l'extrême dans le capitalisme, source de reproduction de rapports de classes, pourrait être considérablement atténuée ${ }^{16}$, en automatisant les travaux répétitifs et en mettant concomitamment l'accent sur la qualification du plus grand nombre.

41 Dans ces conditions, le dépassement du salariat pourrait signifier épanouissement de la subjectivité de chaque individu au travail. Cette subjectivité contribuerait bien sûr à améliorer l'efficience productive moderne, confrontée " aux aléas et aux incidents des flux matériels et immatériels de la production ${ }^{17}$. » Il s'agirait surtout, comme l'exprime si bien Jean-Marie Vincent « de faire sortir les subjectivités des prisons dans lesquelles les enferme la valorisation ${ }^{18}{ }$ et de les ouvrir à la relation multilatérale et à l'enrichissement réciproque.

42 Cette nouvelle subjectivité ne serait pas individualiste, synonyme de choc des ego, de stratégie de valorisation personnelle autiste. Elle serait compatible avec l'intérêt collectif et, plus encore, productrice d'intérêt général. Car selon Jean-Marie Vincent, "les subjectivités doivent s'enrichir réciproquement de la diversité de leurs points de vue, de leurs jugements, de la ressemblance et de la différence de leurs activités dans une perspective d'universalisation qui respecte les singularités et joue sur leurs complémentarités ${ }^{19} »$. 

libéralisme ». La contradiction dommageable pour les mouvements de libération humaine du XXe siècle entre collectivisme et individualisme peut être dépassée.

\section{Comment avancer ?}

Les pouvoirs publics de tendance progressiste, y compris au niveau local, peuvent considérablement favoriser l'émergence de ces formes économiques alternatives, par des mesures réglementaires, par leurs politiques d'achat, par leurs diverses interventions économiques... Pour cela, il faut un peu d'imagination, mais surtout de la volonté et du courage politique. Cela exige de commencer à subvertir les cadres institutionnels étouffants, fixés par le capital. Dans le domaine économique, cette subversion ne sera pas facile, car une multitude de règles rend difficile la transgression. Néanmoins, il est possible de jouer sur les contradictions de ces règles, il est envisageable de détourner des dispositifs. Pour ne citer qu'un exemple, il est imaginable de favoriser fiscalement et socialement, grâce aux dispositifs de zones franches urbaines, des entreprises équitables plutôt que celles qui chassent les primes et exonérations pour augmenter leur taux de profit. Une articulation de plus en plus large peut donc se faire, entre réseaux économiques alternatifs et collectivités locales soucieuses de développement local et social sur leurs territoires, dans une perspective de desserrement des contraintes institutionnelles et marchandes. On peut imaginer la multiplication de petites ou moyennes entreprises sociales et solidaires, développant en commun selon une structure réticulaire des biens et services de complexité croissante. La structure en réseau permet d'allier l'exercice de la citoyenneté économique dans des structures autonomes à effectifs restreints, et des synergies suffisantes, capables de faire face aux grandes structures. Ces réseaux coopératifs d'entreprises sociales et solidaires constitueraient progressivement une alternative économique crédible aux oligopoles, de plus en plus prédateurs. Une voie possible vers le dépassement du salariat...

L'expérience pratique de nouvelles formes économiques ne saurait cependant se substituer totalement aux légitimes luttes syndicales ou politiques, plus classiques. Au contraire, elles pourraient renforcer celles-ci, en montrant pratiquement la possibilité d'alternative ici et maintenant. Dans le même temps, l'affirmation de réseaux économiques équitables solides, affirmant une volonté de dépassement global du salariat, provoquerait un saut qualitatif dans les revendications salariales classiques.

\section{NOTES}

1. Selon le rapport de l'Insee (2003), sur 21,5 millions de salariés en France, 14 millions étaient en CDI à temps plein, auxquels on peut ajouter 4,5 millions de fonctionnaires. NDR.

2. Jean Pélissier, Alain Supiot, Antoine Jeammaud, Droit du travail, Dalloz, 2004, 22e éd.

3. http://perso.orange.fr/marxiens/politic/revenus/richesse.htm

4. Maria Turchetto, Dictionnaire Marx contemporain, PUF, Paris 2001, p. 304. 
5. Michael Hardt, Antonio Negri, Empire, Exils, Paris, 2000, p. 206.

6. La dépense d'énergie est matérielle aussi bien pour produire un bien qu'un service de type logiciel.

7. Nicolas Sarkozy prévoit de supprimer dans les faits le RMI en le reliant à un travail obligatoire pour la collectivité.

8. La tarification de ces prestations doit être différenciée selon le niveau de consommation, afin d'éviter les gâchis.

9. Paul Boccara, «Pistes pour des interventions et scénarios pour une sécurité d'emploi et de formation mobiles dans une régulation nouvelle » in : Issues $n^{\circ} 47-48,1996$.

10. Toutefois, Nicolas Sarkozy en vient maintenant à récupérer en le détournant le terme même de sécurité sociale professionnelle.

11. De nombreux auteurs (notamment Miringoff, Osberg et Sharpe) ont construit des indicateurs illustrant la dissociation progressive entre PIB et bien-être social.

12. Maurice Décaillot, Demain l'économie équitable, L'Harmattan, Paris 2001, p 63.

13. L'existence de travailleurs indépendants demeure possible pour certaines tâches exigeant une division technique relativement faible $\mathrm{du}$ travail. Ces travailleurs indépendants échangeraient avec les autres entités économiques selon des normes équitables.

14. Ces rapports inégalitaires mettant face à face deux échangistes souvent inégaux constituent le fondement de l'échange marchand. Dépasser le marché, c'est construire des échanges équitables entre partenaires économiques.

15. Maurice Décaillot, Au-delà du marché, La Dispute, Paris 1999, pp. 105-116.

16. Nous ne pensons pas que la division hiérarchique du travail puisse être totalement dépassée. Certains individus auront plus de capacité à mener des tâches peu accessibles ou peu souhaitées par l'ensemble des hommes. Pour autant, ces tâches particulières ne doivent plus conférer un pouvoir spécifique d'orientation politique sur la vie économique de l'entreprise ou du collectif de producteurs-associés.

17. Jean-Marie Vincent, «La déstabilisation du travail», in: Futur Antérieur $\mathrm{n}^{\circ}$ 35-36, L'Harmattan, Paris, 1996, p. 17.

18. Ibid., p 28.

19. Ibid.

\section{AUTEUR}

\section{PHILIPPE MAINGAULT}

Enseignant associé à l'Institut d'études européennes de l'Université Paris 8 